\title{
A comparison of HER2 tumor accumulations of Ga- 67 labeled anti-HER2 antibody with chemically and site-specifically conjugated bifunctional chelators
}

\author{
Yumiko Kono ( $\sim$ kohnoy@hirakata.kmu.ac.jp ) \\ Kansai Medical University https://orcid.org/0000-0002-7977-0272 \\ Keita Utsunomiya \\ Kansai Medical University
}

Yuta Ohira

Perseus Proteomics Inc.

Hirokazu Satoh

Perseus Proteomics Inc.

\section{Naoki Kan}

Kansai Medical University

Yohei Matsumoto

Kansai Medical University

Noboru Tanigawa

Kansai Medical University

Original research

Keywords: Drug targets, Site-specific conjugation, Immunotherapy, Radioisotopes, Pharmacokinetics

Posted Date: May 11th, 2020

DOl: https://doi.org/10.21203/rs.3.rs-27077/v1

License: (c) (i) This work is licensed under a Creative Commons Attribution 4.0 International License. Read Full License 


\section{Abstract \\ Background}

Monoclonal antibodies (mAb) developed to target specific cancers have achieved considerable success to-date. To further enhance therapeutic efficacy monoclonal antibodies may be conjugated with a cytotoxic drug or radioisotope. We present the development of new method based on site-specific conjugation (SSC) for targeting HER2. The study design involves a comparison of the accumulation of Ga-67 labeled anti-HER2 antibodies with SSC versus conventional chemical conjugation in HER2-positive tumors. Anti-HER2 antibodies were chemically conjugated (Chem) with the bifunctional chelator deferoxamine (Chem-mAb). The resulting chemical conjugate was radiolabeled with Ga-67 yielding Ga67-Chem -mAb. The SSC anti-HER2 antibodies enzymatically conjugated with deferoxamine using transglutaminase (SSC-mAb) and radiolabeled with Ga-67 yielding Ga-67-SSC-mAb. In vitro, the binding activity of HER2 to both conjugated antibodies was measured using surface Plasmon resonance. In vivo, a xenograft mouse model consisting of transplanted $\mathrm{CHO} / \mathrm{HER} 2$ were divided into two groups, the Chem and the SSC group. Planar images were acquired over three days after each mAb injection, respectively. Pharmacokinetic analysis was used to compare the Chem group to the SSC group, for Ga-67 accumulation.

\section{Result}

The SSC and Chem groups were found to have similar HER2 binding capacity, however the tumor accumulation ratio gradually increased in the SSC group. The pharmacokinetic studies also found that radiolabeled $\mathrm{mAb}$ accumulation was significantly higher in the SSC group than the Chem group in not only the tumors, but also in blood and in other organs.

\section{Conclusion}

The new site-specific conjugation may improve targeted antigen-specific cancer radioimmunotherapy and may, due to higher retention, require a lower dose.

\section{Background}

Monoclonal antibodies that bind to specific tumor surface antigens have demonstrated their potential as effective therapeutics in oncology. However, many naked monoclonal antibodies have limited therapeutic activity (1). Combining the specificity and selectivity of a monoclonal antibody to the efficacy of a potent chemotherapeutic agent or radioisotope is an alluring prospect for targeted location-specific treatment of cancer. Such antibody-drug and antibody-radioisotope conjugates are produced by chemically linking a cysteine or lysine monoclonal antibody $(\mathrm{mAb})$ residue to potent chemotherapeutic agents or radioisotopes by means of a variety of linkers $(2-4)$. However, researchers have found that conventional 
conjugation methods yield heterogeneous molecules $(5,6)$. Antibody-drug conjugates composed of a chemotherapeutic agent plus $m A b$ have been found to have limited efficacy due to their binding site heterogeneity. Recent studies have shown that the new site-specific conjugation methods; can produce binding-site uniform antibody-drug conjugates (Fig. 1) (7). The binding-site homogeneity of antibodydrug/radioisotope conjugates is expected to provide better therapeutic efficacy than previous conventional methods.

The main objective of this paper is to evaluate whether the site-specific conjugation exhibits better biodistribution in HER2-positive tumors, compared to conventional conjugation, when an anti-HER2 antibody is labeled with Ga-67.

\section{Methods}

\section{Antibody preparation}

The anti-human Her2 monoclonal antibody, TSPZ01, was originally derived from the trastuzumab amino acid sequence. To facilitate site-specific bacterial transglutaminase-mediated conjugation of cadaverinederivatized chelator (deferoxamine) to the GIn-residue at position 295 of the heavy chain, the Asp-residue at position 297 of the TSPZ01 heavy chain was replaced with an Ala-residue (N297A). The synthetic cDNA encoded TSPZ01 heavy chain and light chains were inserted into a mammalian expression plasmid.

For antibody production, HEK293 were transiently transfected with the expression plasmid using ExpiFectamine $^{\mathrm{TM}} 293$ reagent (Thermo-Fisher Scientific) following the protocol of the HEK 293 Expression System (Invitrogen). At 6 days post transfection, the culture supernatant was harvested, and the antibody was purified by binding to Protein A (ProteNova; $A b$-Capcher ExTra ${ }^{T M}$ ) $=$ following the manufacture's protocol.

\section{Expression and purification of recombinant Her2 ECD antigen}

A synthetic cDNA fragment encoding the extra cellular domain of human Her2 (amino acid residues 1652) followed by a Histidine tag (RGSHHHHHHHH) at its C-terminus was inserted into a vector for mammalian expression ( $\mathrm{pCXN}$ ). 293-F cells were transfected with the resultant plasmid and culture sup was harvested. The expressed Her2 ECD antigen was purified by binding to HisTrap HP (GE Healthcare) following the manufacturer's instructions. Her2 ECD was eluted from the HisTrap HP with $500 \mathrm{mM}$ imidazole in Dulbecco's PBS, then dialyzed from Dulbecco's PBS, and stored at $-80^{\circ} \mathrm{C}$ until use.

\section{Conjugation of the chelator to $\mathrm{mAb}$}

For chemically conjugation, the TSPZO1 in $\mathrm{NaHCO} 3(\mathrm{pH} 9.0)$ was concentrated using Amicon 10K (Merck Millipore), then adjusted to $10 \mathrm{mg} / \mathrm{ml}$. The bifunctional chelator pSCN-Bn-Deferoxamine (DFO) (Macrocyclics) was dissolved in DMSO, added to the TSPZ01 solution in a 2.3:1 ratio. After 2 hours of 
incubation at $37^{\circ} \mathrm{C}$, the DFO covalently conjugated TSPZ01 (DFO-Chem-TSPZ01, Chem-Ab) was purified using a NAP-5 column (GE healthcare) to remove the free pSCN-Bn-DFO.

For site-specific conjugation, the TSPZ01 in potassium-free PBS (pH8.0) was concentrated using the same method as above and adjusted to $2 \mathrm{mg} / \mathrm{ml}$. The chelator DFO mesylate salt (SIGMA-Aldrich) was dissolved in potassium-free PBS (pH8.0), added to the TSPZ01 solution in a 300:1 ratio. The TSPZ01 and DFO mixture was incubated overnight at $37^{\circ} \mathrm{C}$ with $0.1 \mathrm{U} / \mathrm{uL}$ of recombinant microbial transglutaminase (TGase) (Zedira). To separate the DFO enzymatically conjugated TSPZ01 (DFO-SSC-TSPZ01, SSC-Ab) from free DFO and TGase, buffer exchange was repeated for five times using an Amicon 50K (Merck Millipore)

\section{Calculation of chelator antibody ratio (CAR)}

The calculation of CAR was done using a partially modified method which has been previously described(8). Briefly, deferoxamine solutions for the standard curve were adjusted to $198,132,88,59,39$, $26 \mu \mathrm{M}$ in $0.25 \mathrm{M} \mathrm{NaOAc}$ pH5.5 and antibody sample was adjusted to $9 \mathrm{mg} / \mathrm{mL}(60 \mu \mathrm{M}) .225 \mu \mathrm{L}$ of sample or standard was added to 96 wells in a plate followed by the addition of $25 \mu \mathrm{L}$ of $50 \mathrm{mM} \mathrm{Fe}$ (II) to each well. After the plate was shaken at $700 \mathrm{rpm}$ for 1 hour at room temperature, the absorbance at $450 \mathrm{~nm}$ were measured and the concentration of DFO in sample was calculated. CAR was calculated using the following equation:

$C A R=\frac{\text { CaluculatedDFOconc. }(\mu M)}{\text { antibodyconc. }(60 \mu M)}$

\section{Binding affinity measurements}

Real-time binding analysis was performed at $25^{\circ} \mathrm{C}$ using an SPR biosensor, Biacore 8K system (GE Healthcare). For immobilization of each antibody on the surface of a sensor chip, an anti-human IgG antibody (Human Antibody Capture Kit, GE Healthcare) was first attached to a CM5 sensor chip. The antihuman IgG antibody was diluted to $25 \mu \mathrm{g} / \mathrm{mL}$ in immobilization buffer (10 mM sodium acetate; $\mathrm{pH} 5.0$ ) and coupled to the carboxymethylated dextran surface of a CM5 sensor chip using a NHS/EDC coupling procedure. The amount of immobilized protein was approximately 10,000 resonance units (RU). Next, antibodies were diluted to $0.65 \mu \mathrm{g} / \mathrm{ml}$ in running buffer (HBS-EP+ buffer (10 mM HEPES; pH 7.4; $150 \mathrm{mM}$ $\mathrm{NaCl} ; 3 \mathrm{mM}$ EDTA; $0.05 \%$ surfactant P20)) and then injected into each flow cell until the amount reached approximately $100 \mathrm{RU}$. The flow rate of the running buffer for protein interaction was maintained at 10 $\mu \mathrm{L} / \mathrm{min}$. A dilution series of Her2 ECD solution was injected into the running buffer ( $50 \mathrm{nM}, 16.7 \mathrm{nM}, 5.6$ $\mathrm{nM}, 1.9 \mathrm{nM}, 0.6 \mathrm{nM}$ ) in single-cycle kinetics mode, flowing over the immobilized sensor surface for 180 $\mathrm{sec}$ at each concentration. Afterwards the sensor surface was washed with running buffer for $600 \mathrm{~s}$. The sensor surface was regenerated by regeneration solution ( $3 \mathrm{M}$ magnesium chloride) injected foe $30 \mathrm{sec}$ at a flow rate of $20 \mu \mathrm{L} / \mathrm{min}$. The equilibrium dissociation constant (KD) value for the binding affinity was calculated using a 1:1 binding model. 


\section{Capillary isoelectric focusing analysis}

The capillary isoelectric focusing (CIEF) analyses were carried out using an iCE280 system (ProteinSimple). Monoclonal antibody samples in $20 \mathrm{mM}$ Na-phosphate $\mathrm{pH} 6.0$ were prepared using a ZebaSpin desalting column (ThermoFisher). The sample was prepared with $4 \%$ Pharmalytes $3-10,0.35 \%$ methylcellulose, and $2 \mathrm{M}$ urea, $0.5 \%$ of each pl marker (4.22 and 9.46 ), and $140 \mu \mathrm{g} / \mathrm{mL}$ of Naked mAb or $400 \mu \mathrm{g} / \mathrm{mL}$ of DFO conjugated antibody. These cIEF analysis samples were gently mixed, briefly centrifuged, and immediately processed by CIEF according to the manufacture's protocol. The resulting data were analyzed using the iCE280 CFR and Chrome Perfect (ProteinSimple) software.

\section{Radiolabeling of DFO conjugated TSPZ01 with Ga-67}

Chloride-Ga-67 was kindly donated from Fujifilm RI Pharma Co., Ltd. The DFO conjugated TSPZ01 antibodies were radiolabeled with Ga-67 (73.8 MBq / mL, $12.3 \mathrm{MBq} / \mathrm{mg}$ ) in 0.25 M NaOAc (pH5.5) buffer for $60 \mathrm{~min}$ at $45^{\circ} \mathrm{C}$. After the labeling yields were confirmed to be at least $95 \%$, each sample was purified using a desalting column (ThermoFisher). The concentration of antibody and the amount of radioactivity were calculated using a NanoDrop 1000 (Thermo Fisher) and Curie Meter IGC-8 (Hitachi), respectively.

\section{Serum stability assay}

The Chem-mAb and SSC-mAb labeled with Ga- 67 were added, at concentration of $15 \mu \mathrm{g} / \mathrm{mL}$, to human serum. The labeling yields were measured, to evaluate the stabilization in coupling between antibody and Ga-67, at day 0, day 1, day 4, and day 7 using instant thin-layer chromatography (iTLC). The sample radioactivity was counted using an automatic gamma counter (WIZARD ${ }^{\text {TM }} 3^{\text {" }} 1480$, Perkin Elmer, Life Science). The binding ratios of the Chem mAb and the SSC mAb were measured. The serum stability assay was repeated four times.

\section{Ga-67 imaging in vivo study}

Approximately 5 million cells of Her2 over-expressed Chinese hamster ovary (Her2/CHO) were subcutaneously inoculated into a BALB/c-nu/nu mouse model ( $7 w$, female) at the dorsal right shoulder. The tumors were grown for one week. Fourteen such mice were prepared for Chem-mAb injected mice (Chem group, $n=7$ ) and SSC-mAb injected mice (SSC group, n=7). Planar scanning was performed with the Small-Animal Imaging System (Inveon; Siemens Medical Solutions). Thirty $\mu \mathrm{g}$ of each antibody and Ga-67 (370 kBq) was injected into the mice tail veins at $100 \mu \mathrm{L}$ per mouse. After each antibody injection, planar images were taken every day from day 1 to day 3 . The accumulation rate (AR) was defined as the ratio of the tumor region of interest, $R O I_{\text {Tumon }}$ normalized by a whole-body region of interest, $R O I_{W B}$, thus

$$
A R=\frac{R O I_{\text {Tumor }}}{R O I_{W B}}
$$


Specifically, $R O I_{\text {Tumor }}$ was defined as a circular region, with a diameter of $6 \mathrm{~mm}$, at the tumor mass of the irradiated site and $R O I_{W B}$ was defined as a region, with a rectangle included the whole body of mouse, The ARs of SSC group were compared to the Chem group. In addition, SPECT images were acquired for identification of the accumulation sites at day 2 after antibody injection.

\section{Biodistribution study}

At 4 day after Chem-Ab and SSC-Ab injections, all the animals were sacrificed and then dissected. The Her2/CHO tumor and major organs were extracted and weighed, then the radioactivity count was measured using an automatic gamma counter (WIZARDTM 3" 1480, Perkin Elmer, Life Science). The resulting data were expressed as the percentage of injected dose per gram of tissue (\% ID/g).

\section{Statistical analysis}

The statistical analysis of the results of the in vitro serum stability test was performed using repeated ANOVA. The in vivo planar imaging and pharmacokinetics of Ga-67 accumulation studies statistical analyses were performed using the Mann-Whitney U-test. A threshold for statistical significance for all statistical analyses was set as $p<0.05$.

\section{Results}

\section{Chemical and enzymatically modification of antibodies}

TSPZ01 mAb was conjugated with the Deferoxamine bifunctional chelating agent using both chemical and enzymatic methods. In the chemical conjugation method, the pSCN-Bn-Deferoxamine was covalently bound to the Lys-residues of TSPZ01. In the enzymatic conjugation method, the Deferoxamine mesylate salt was enzymatically reacted with the Gln-residue at position 295 of the TSPZ01 heavy chain with Transglutaminase. The chelator antibody ratio (CAR) for chemical conjugation was 1.76 , and 1.83 for the enzymatic conjugation. These results indicate that the CAR is comparable between the chemical and the enzymatic conjugation methods.

The heterogeneity of the chelator-modified Chem mAb and SSC mAb antibodies was assessed using cIEF. Chem mAb exhibited broader charge heterogeneity than TSPZ01 (naked antibody), suggesting that DFOantibody conjugation had occurred at random positions and in random number (Fig.1). On the other hand, SSC-mAb exhibited similar broad heterogeneity relative to TSPZ01, contrary to the expectation that DFO-antibody conjugation binding would be site-specific with fixed number.

To examine the effect of chelator conjugation on antibody to Her2 binding activity, we performed a SPR biosensor kinetic interaction analysis - with naked mAb, Chem-mAb, and SSC-mAb immobilized on the sensor chip surface, respectively. The dissociation constants (KD) were estimated to be approximately $0.8 \mathrm{nM}$ for Chem-Ab and $1.0 \mathrm{nM}$ for SSC-mAb, respectively. The Chem-mAb and SSC-mAb KD values 
were found to be comparable and both were comparable to the naked mAb, TSPZ01, value (Supplemental Table). These data indicated that there is no significant difference in the binding activity between Chem-mAb and SSC-mAb and that both chemical and enzymatic conjugation did not significantly affect the naked mAb binding activity.

One potential confounding factor regarding binding activity is Ga-67-chelator-mAb disconjugation in human serum yielding naked $\mathrm{mAb}$ and chelator-Ga-67; confounding the dissociation constant measurements and thereby yielding similar binding activities to naked $\mathrm{mAb}$. We therefore carried out a serum stability study. The Ga-67 labeling yields of Chem-mAb and SSC-mAb decreased to about $85 \%$ after one week (Fig.3). SSC-mAb has almost the same retention of Ga-67 as Chem-mAb. This trend was observed with both conjugated antibodies; there was no significant difference in chelator-antibody disconjugation.

\section{Imaging and biodistribution in vivo study}

Using planar imaging, the ARs of the two animal groups, Chem-mAb-Ga-67 and SSC-mAb-Ga-67 injected, were calculated from day 1 to day 3 post-injection (Fig.4). The AR of both groups increased day by day. However, the SSC-mAb-Ga-67 group's AR increased more than the Chem-mAb-Ga-67 group's AR and a significant difference was observed between the AR of Chem group and SSC group at day 3

Using SPECT imaging, a higher accumulation of Ga-67 was observed at the tumor site in SSC-mAb-Ga67 mouse group than in the Chem-mAb-Ga-67 group (Fig.6).

The biodistribution study found, at the tumor site, a significantly higher accumulation of SSC-mAb-Ga$67,20.4 \pm 4.19 \% \mathrm{ID} / \mathrm{g}$ (mean $\pm \mathrm{SD}, \mathrm{n}=6$ ), than Chem-mAb-Ga-67, $11.7 \pm 2.95 \% \mathrm{ID} / \mathrm{g}($ mean $\pm \mathrm{SD}, \mathrm{n}=7)$ $(P=0.010 ;$ Fig. 5). However, similar accumulation trends were observed in other organs. For example, there was a statistically significant difference between the SSC-mAb-Ga-67 group uptake in blood, $8.16 \pm$ $0.76 \% \mathrm{ID} / \mathrm{g}$ (mean $\pm \mathrm{SD}, \mathrm{n}=6$ ), compared to the Chem-mAb-Ga-67 group, $5.78 \pm 1.39 \% \mathrm{ID} / \mathrm{g}(\mathrm{n}=7)$; also SSC-mAb-Ga-67 group uptake in liver $17.42 \pm 2.01 \% \mathrm{ID} / \mathrm{g}(\mathrm{n}=6)$, compared to the Chem-mAb-Ga-67 group, $11.08 \pm 3.64 \% \mathrm{ID} / \mathrm{g}(\mathrm{n}=7)(\mathrm{P}=0.014,0.024)$.

\section{Discussion}

The purpose of this study was to compare the accumulation, in HER2-positive tumors, of Ga-67 labeled anti-HER2 monoclonal antibodies with site-specific conjugation versus conventional chemical conjugation.

In conventional conjugation, the pSCN-Bn-deferoxamine can be accessible to surface Lysine residues on the antibody molecule. Therefore, the pSCN-Bn-deferoxamine binds randomly to primary amine sites, possibly binding to Lysine residues in the antigen-binding region, which is the complementarity determining region (CDR). This work is to clarify (i) whether binding on Lysine residues on the CDR or 
their vicinity of antibody interfere the affinity that is functional binding to target antigen, (ii) the optimal molar ratio of $\mathrm{pSCN}$ - Bn-deferoxamine to antibody.

A previous study reported that the number of drug molecules that can be conjugated to one antibody without affecting antigen binding was limited (9). As conventional chemical conjugation may modify the antigen-binding region on an antibody, we hypothesized that site specific conjugation, yielding, SSC-mAb, may exhibit superior binding to antigen relative to Chem-mAb in in vitro studies. However, no significant difference between the Chem-mAb and SSC-mAb KD values was observed. To account for this observation, we postulate that the antigen binding regions of Chem-mAb are rarely modified in a low chelator - antibody ratio environment.

When the CAR is less, the conjugated antibody has not enough therapeutic efficacy against tumor cells. On the other hand, when the CAR is much more than optimal, the chelators may cause aggregation of the antibody due to hydrophobic bond may tend to break hydrated state of antibody. Thus, the more sitespecific conjugation becomes recent trend, the more such information what the CAR is required for best therapeutic design, eg. how many chelators is the best under in vivo study.

Although no significant difference was found between the SSC-mAb and Chem-mAb KD values, on the other hand, the large difference in electric charge heterogeneity was observed. A previous study reported that the positional isomer variant, resulting from chemical conjugation, likely account for the high charge heterogeneity (9). Such charge variants exhibit markedly different colloidal stabilities, blood stabilities, solubilities, pharmacokinetics, and tissue distributions, notably tumor accumulation, making quality control major problem in manufacturing. $(10,11)$. The larger the variation in the electric charge distribution, the more difficult it is to predict the biodistribution, raising the possibility of unexpected side effects. These results suggested that site-specific conjugation maybe advantageous for quality control during production and expected efficacy.

Chem-mAb and SSC-mAb exhibited similar stability in blood serum. As the bifunctional chelator, deferoxamine, used for both chemical conjugation (Chem) and site-specific conjugation (SSC) is the same and as the difference in affinity between the chelator and Ga-67 is negligible in both mAbs, the similar KD values of Chem-mAb and SSC-mAb suggested that the chelator - mAb bond, formed by the thiocyanate and the primary amine (Chem) and by the transglutaminase (SSC), have similar stability in serum.

Our in vivo animal model studies have indicated higher tumor accumulation in SSC-mAb than ChemmAb. These results suggest higher uptake in other organs, notably the liver and spleen, may be accounted for by higher SSC-mAb stability in in vivo whole blood, unlike our in vitro blood serum study. Further investigation is required to understand this observed difference between the in vivo and in vitro results.

Monoclonal antibodies are large $(150 \mathrm{kDa})$ multimeric proteins that contain numerous disulfide bonds and post-translational modifications such as glycosylation. The mAbs are characterized by a very long serum half-life as they are far above the renal clearance threshold (70 kDa) and are thus blocked from 
being cleared through the renal glomerulus (12). In murine xenograft models, monoclonal antibodies directed against tumor-specific antigens still largely remain in the blood. Only $20 \%$ or less of the administered dose interacts with the tumor (13). According to biodistribution studies in animal models lacking the target antigen, the largest dose percentage of monoclonal Ab remains in the plasma and thus whole-body distribution predominantly targets organs that are highly perfused with blood $(14,15)$.

In our in-vivo study, the site-specific conjugated monoclonal antibody, SSC-mAb, exhibited a longer retention time in blood then Chem-mAb. It was exposed to more SSC-mAb over the same time that the SSC-mAb organ accumulation was higher because highly perfused organs, such as the liver and spleen. This difference may be related to the true homogeneity of the antibody. However, our biodistribution data indicates that the liver-to-blood ratio and spleen-to-blood ratio were higher in the SSC-mAb group than in the Chem-mAb group, suggesting that other factors contribute besides the blood concentration factor. Further work is required to elucidate what other factors may be contributing to the observed difference in these ratios.

Further investigation is required to uncover the mechanism of enhanced of Chem-mAb-Ga-67 and SSCmAb-Ga-67 accumulation in the liver and spleen. We have found that a potential advantage of the site specific conjugated monoclonal antibody is higher accumulation in the target HER2 tumor relative to the conventional chemically conjugated monoclonal antibody. As higher stability in blood of SSC Ab, it could reduce the cost of anti-body therapy through a reduction in injection dose of antibody.

\section{Conclusions}

The site-specific conjugation method enhanced the accumulation of anti-HER2 monoclonal antibody labeled with Ga-67 in HER2 tumors. Meanwhile, increased accumulation in non-target organs, notably the liver and spleen, was also observed.

\section{Abbreviations}

mAb

Monoclonal antibodies

SSC

site-specific conjugation

HER2

human epidermal growth factor receptor 2

$\mathrm{CHO}$

Chinese hamster ovary

CAR

Calculation of chelator antibody ratio

iTLC

instant thin-layer chromatography 


\section{Declarations}

\section{Ethical Approval and Consent to participate}

This animal study obtained approval from an appropriate animal care committee in conformance with the Guide for the Care and Use of Laboratory Animals (Institute for Laboratory Animal Research (ILAR). 8th Edition, 2010).

\section{Consent for publication}

Not applicable.

\section{Availability of data and materials}

The datasets used and analyzed during the current study are available from the corresponding author on reasonable request.

\section{Competing interests}

The authors declare that they have no competing interests.

\section{Funding}

This work was supported by the research grant D2 from Kansai Medical University.

\section{Authors' contributions}

YK was involved in the study design and the acquisition, analysis, and interpretation of all data. YO and HS helped design the study and acquired and analyzed in vitro data. $\mathrm{KN}$ and $\mathrm{YM}$ were involved in vivo experiments and their analysis and interpretation. KU and NT were involved in study design, analysis, interpretation of all data and results and wrote the manuscript. All authors read and improved the final manuscript.

\section{Corresponding author}

Correspondence to Yumiko Kono.

\section{Authors' information}

\section{Affiliations}

Department of Radiology, Kansai Medical University, Osaka, Japan

Yumiko Kono, Keita Utsunomiya, , Naoki Kan, $₫$ Noboru Tanigawa ${ }^{1}$

Perseus Proteomics Inc. 
Yuta Ohira $\otimes$ Hirokazu Satoh ${ }^{2}$

RadioisotopeResearch Center, Kansai Medical University, Osaka, Japan

Yohei Matsumoto

\section{Acknowledgments}

The authors thank Audrius Stundzia, Ph.D., Tomographix IP Ltd., for valuable advice about this article, and revision of the draft manuscript, based on detailed discussion and feedback from all the authors; this assistance was funded by Gakunai Josei D2 in Kansai Medical University. This work was partially supported by the Platform Project for Supporting Drug Discovery and Life Science Research from Japan Agency for Medical Research and Development (AMED)

\section{References}

1. Wu AM, Senter PD. Arming antibodies: prospects and challenges for immunoconjugates. Nature biotechnology. 2005;23(9):1137-46.

2. Carter PJ, Senter PD. Antibody-drug conjugates for cancer therapy. Cancer journal. 2008;14(3):15469.

3. McCarron PA, Olwill SA, Marouf WM, Buick RJ, Walker B, Scott CJ. Antibody conjugates and therapeutic strategies. Mol Interv. 2005;5(6):368-80.

4. Polakis P. Arming antibodies for cancer therapy. Curr Opin Pharmacol. 2005;5(4):382-7.

5. Hamblett KJ, Senter PD, Chace DF, Sun MM, Lenox J, Cerveny CG, et al. Effects of drug loading on the antitumor activity of a monoclonal antibody drug conjugate. Clinical cancer research: an official journal of the American Association for Cancer Research. 2004;10(20):7063-70.

6. Wang L, Amphlett G, Blattler WA, Lambert JM, Zhang W. Structural characterization of the maytansinoid-monoclonal antibody immunoconjugate, huN901-DM1, by mass spectrometry. Protein science: a publication of the Protein Society. 2005;14(9):2436-46.

7. Jager M, Nir E, Weiss S. Site-specific labeling of proteins for single-molecule FRET by combining chemical and enzymatic modification. Protein science: a publication of the Protein Society. 2006;15(3):640-6.

8. Saywell LG, Cunningham BB. Determination of Iron: Colorimetric o-Phenanthroline Method. Industrial Engineering Chemistry Analytical Edition. 1937;9(2):67-9.

9. Chari RV. Targeted delivery of chemotherapeutics: tumor-activated prodrug therapy. Adv Drug Deliv Rev. 1998;31(1-2):89-104.

10. Boylan NJ, Zhou W, Proos RJ, Tolbert TJ, Wolfe JL, Laurence JS. Conjugation site heterogeneity causes variable electrostatic properties in Fc conjugates. Bioconjugate chemistry. 2013;24(6):100816. 
11. Lhospice F, Bregeon D, Belmant C, Dennler P, Chiotellis A, Fischer E, et al. Site-Specific Conjugation of Monomethyl Auristatin E to Anti-CD30 Antibodies Improves Their Pharmacokinetics and Therapeutic Index in Rodent Models. Mol Pharm. 2015;12(6):1863-71.

12. Chames $P$, Van Regenmortel M, Weiss E, Baty D. Therapeutic antibodies: successes, limitations and hopes for the future. Br J Pharmacol. 2009;157(2):220-33.

13. Holliger P, Hudson PJ. Engineered antibody fragments and the rise of single domains. Nature biotechnology. 2005;23(9):1126-36.

14. Lin YS, Nguyen C, Mendoza JL, Escandon E, Fei D, Meng YG, et al. Preclinical pharmacokinetics, interspecies scaling, and tissue distribution of a humanized monoclonal antibody against vascular endothelial growth factor. J Pharmacol Exp Ther. 1999;288(1):371-8.

15. Fox JA, Hotaling TE, Struble C, Ruppel J, Bates DJ, Schoenhoff MB. Tissue distribution and complex formation with $\lg E$ of an anti-lgE antibody after intravenous administration in cynomolgus monkeys. J Pharmacol Exp Ther. 1996;279(2):1000-8.

\section{Figures}

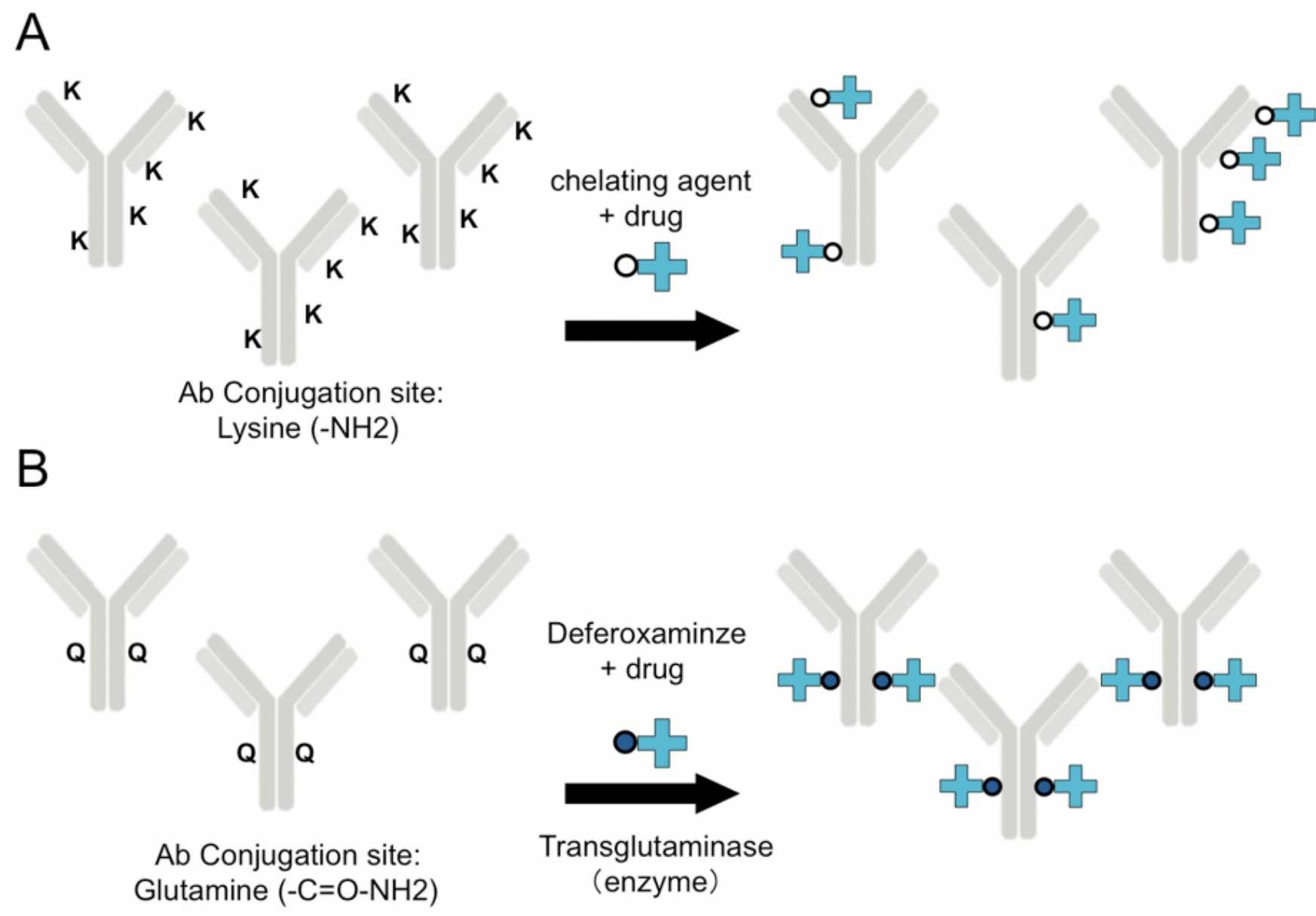




\section{Figure 1}

The difference between chemical conjugation and site-specific conjugation methods. (A) The conventional chemical reaction give rise to heterogeneous conjugates, which lack site-specificity, linkage stability, and stoichiometric control and may cause poor in vivo performance. (B) New methods of sitespecific conjugation form homogeneous molecules with uniform efficacy and greater stability, which may lead to improved therapeutic efficacy relative to past conventional chemical conjugation methods.

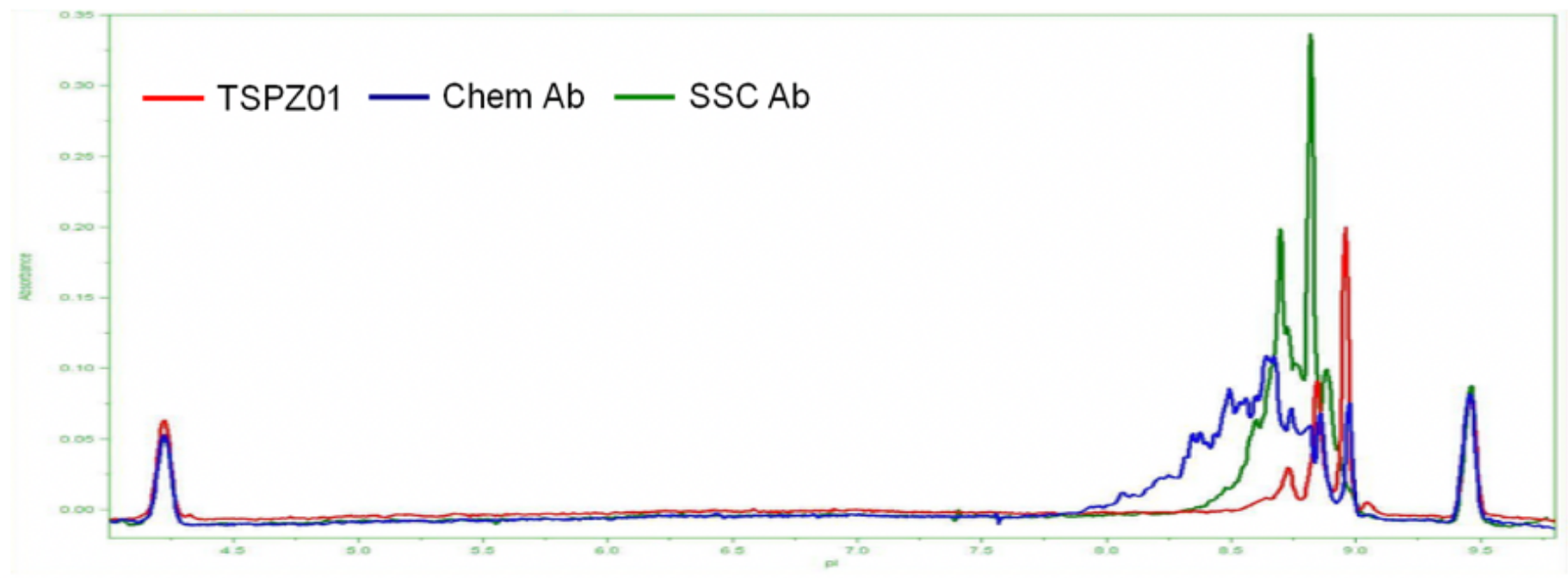

Figure 2

Charge heterogeneity profile of antibodies. Naked and modified antibody was subjected to cIEF analysis. Each sample was electrophoresed with pl marker and then overlaid. 


\section{Binding ratio}

(\%)

100

90

80

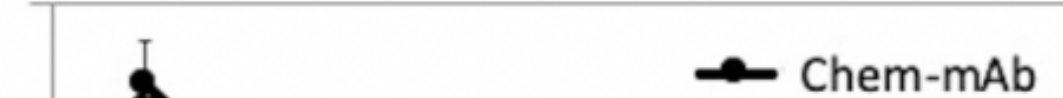
$-\mathrm{Ga}-67$

SSC-mAb

$-\mathrm{Ga}-67$

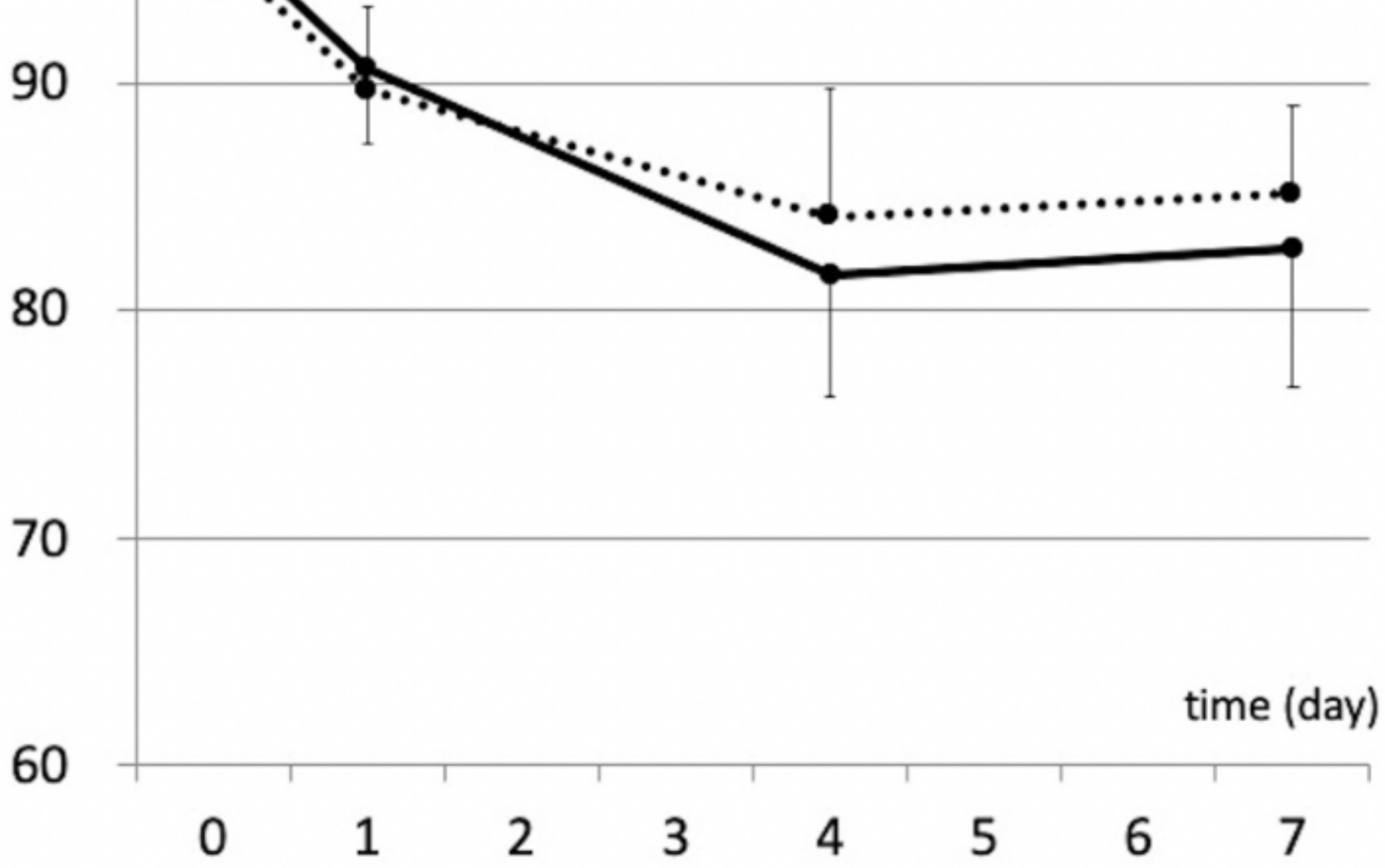

Figure 3

Comparison of Chem and SSC samples in a serum stability study Chem-mAb and SSC-mAb were incubated with human serum over 7 days. Each sample was measured by iTLC and averages of 4 tests were plotted. Error bars are expressed as mean (SD). These two samples exhibited no difference in stability following incubation with human blood serum. 
A

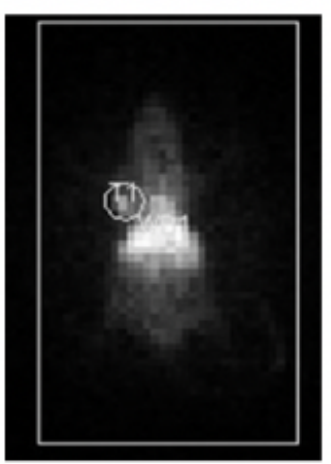

$\mathrm{B}$

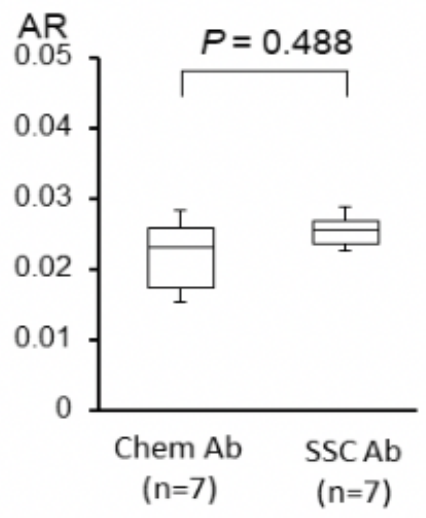

C

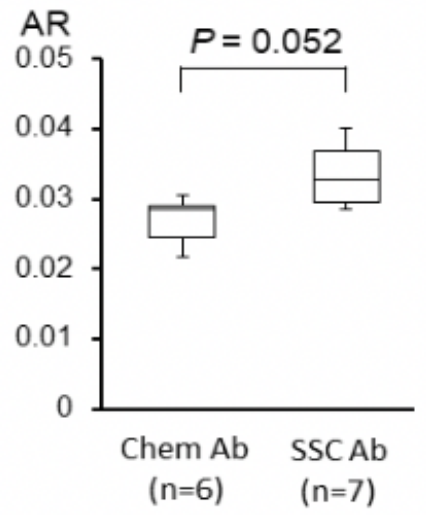

D

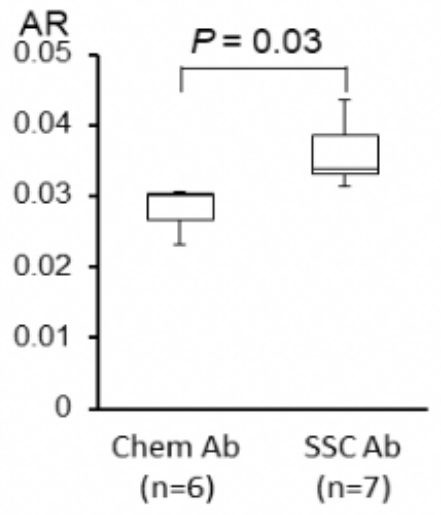

\section{Figure 4}

Accumulation rate measured by planar imaging. $\mathrm{ROI}$ selection and AR calculation by Ga- 67 planar imaging. ROls are drawn around entire tumor and whole body. (B) Day1, (C) day2, and (D) day3 after intravenous injection of Ga-67-Chem-mAb or Ga-67-SSC-mAb. Significance testing was done using the pairwise Mann-Whitney $U$ test. One mouse from the Chem group died due to excessive anesthesia on the 2nd day. Significant difference between Ga-67-Chem-mAb and Ga-67-SSC-mAb accumulation was observed at 3rd day after injection. Error bars are expressed as mean (SD), $n=6-7$. 


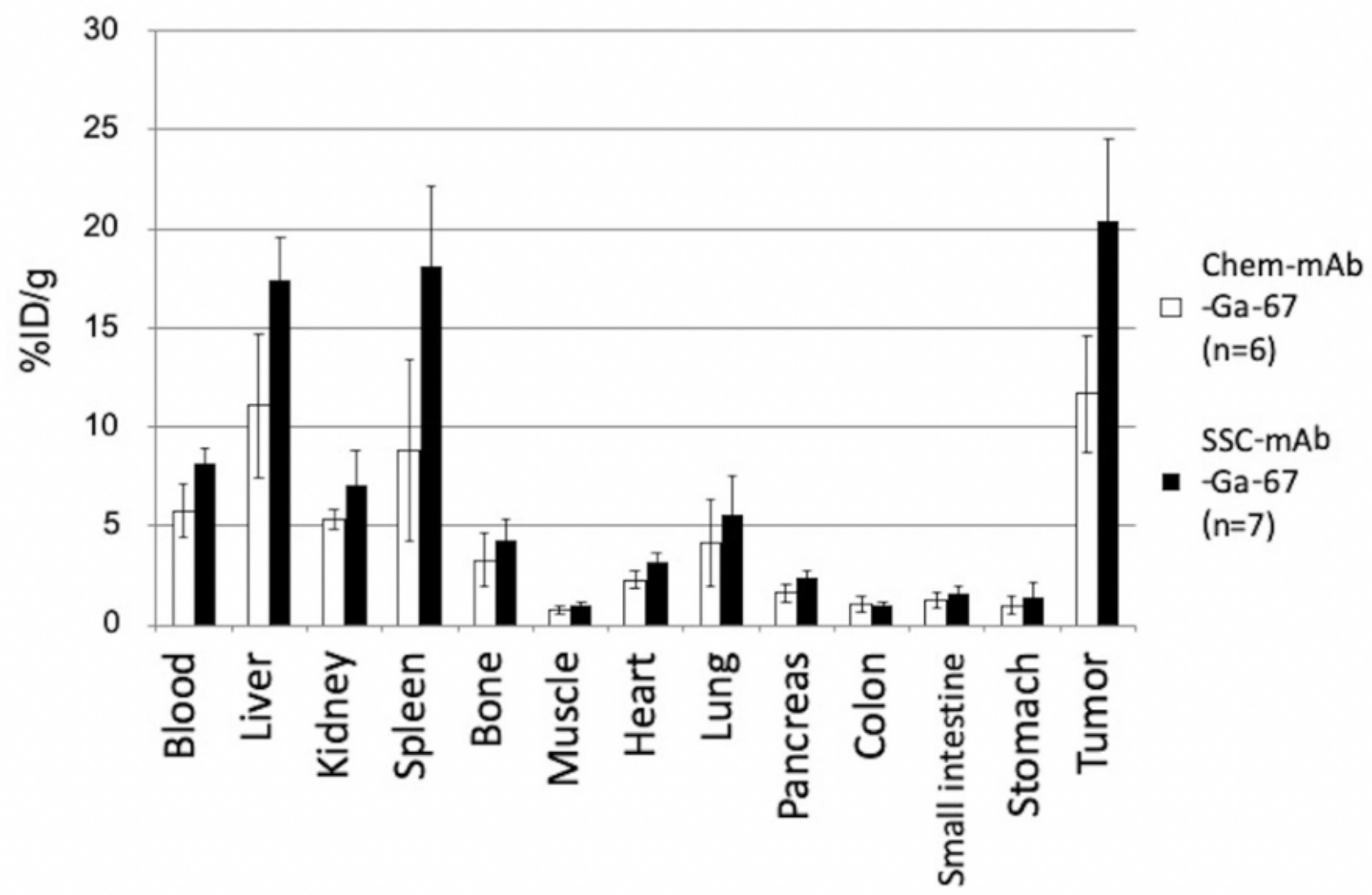

Figure 5

Biodistribution of $\mathrm{Ga}-67$ in Her2/CHO tumor model mice. Tumors and organs were collected 96 hours after intravenous injection of each Ga-67-Chem-mAb or Ga-67-SSC-mAb into the tail vein. Probe distribution was determined by radioactivity and analyzed \%ID/g, ex vivo. ${ }^{*} P<0.05$. Error bars expressed as mean (SD). 


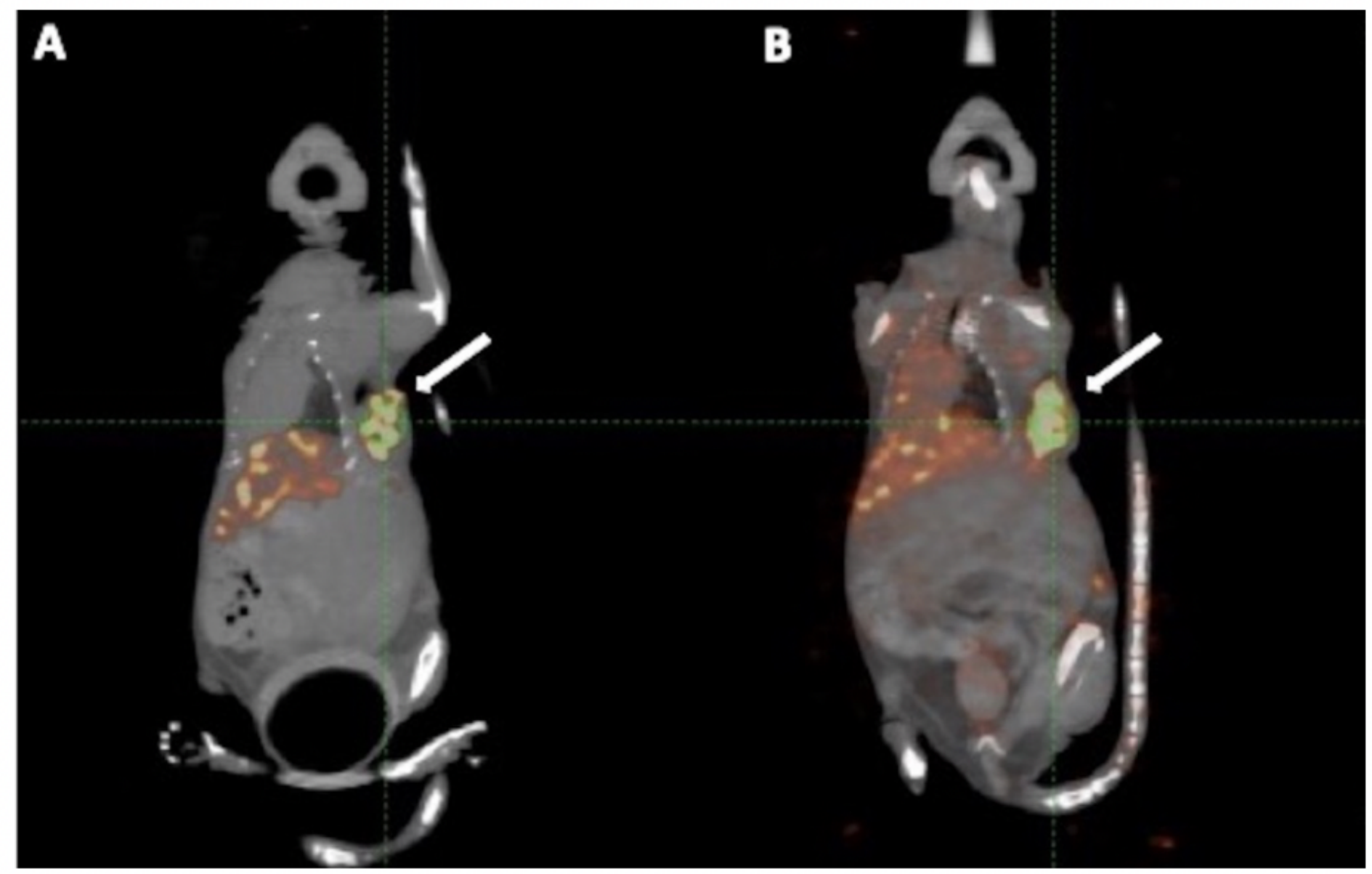

\section{Figure 6}

SPECT/CT imaging. (A) Ga-67-Chem-mAb injected mouse. (B) Ga-67-SSC-mAb injected mouse. SPECT images were showed at the same window width and window level in digital image. More intense activity is seen in the SSC mouse tumor than in the Chem group (white arrow). Similarly, background accumulation was also higher in the SSC group than Chem group.

\section{Supplementary Files}

This is a list of supplementary files associated with this preprint. Click to download.

- Supplementaltable1.docx 